\title{
Factors affecting rats' location during conditioned suppression training
}

\author{
RICK A. BEVINS and JOHN J. B. AYRES \\ University of Massachusetts, Amherst, Massachusetts
}

\begin{abstract}
If freezing underlies barpress conditioned suppression, then it seems odd that auditory cues paired with shock evoke more freezing than do visual cues, yet evoke similar suppression. Bevins and Ayres (1992) found that auditory and visual cues also evoked similar withdrawal from the bar and dipper areas and suggested that such withdrawal could explain the similar suppression. Seeking to understand that withdrawal, we found evidence in the present study that it was due either to adventitious punishment or to place-aversion learning. The cue for shock seemed to set the occasion for such learning. For example, we found that, as training progressed, rats' tendency to leave the bar area during the cue first increased, then decreased, then increased again, reflecting, presumably, shock occurrence first inside, then outside, then inside the bar area again. Despite these changes in the rats' location, barpress suppression remained stable, implying that leaving the bar area, though sufficient for barpress suppression, is unnecessary.
\end{abstract}

Conditioned stimuli (CSs) that precede shock unconditioned stimuli (USs) suppress appetitively reinforced responding in rats (Estes \& Skinner, 1941). Such CSs also evoke freezing, which is defined as lack of movement except for that of the rat's sides, as required for breathing (Fanselow \& Bolles, 1979). Because freezing functions as a successful antipredator behavior (Fanselow \& Lester, 1988), competition between the elicited defensive behavior and the appetitive operant offers an attractive explanation for conditioned barpress suppression; indeed, freezing and suppression often are highly correlated (Ayres \& Vigorito, 1984 , Bouton \& Bolles, 1980). However, if freezing were solely responsible for conditioned suppression, CSs evoking different levels of freezing should evoke corresponding differences in barpress suppression, which is not the case.

Auditory CSs, such as white noise or tone, often evoke more freezing than do visual CSs, such as increases or decreases in light, but simultaneously evoke a similar level of barpress suppression (Ayres, Axelrod, Mercker, Muchnik, \& Vigorito, 1985; Bevins \& Ayres, 1991). Bevins and Ayres (1992) found that a tone or light paired in a forward manner with shock evoked movement away from the area of the operant box containing the response bar and reinforcement dipper, causing the rats to enter the

This research was supported by Grant BNS 87-23173 from the National Science Foundation and by a Faculty Research Grant from the University of Massachusetts to J.J.B.A. We thank Melinda Novak for comments on an earlier draft of the manuscript and Stephen Kim for help in running parts of Experiment 2, including checking reliability of observations. R. Bevins is now at Kastle Hall, University of Kentucky, Lexington, KY 40506. Correspondence may be addressed to J. J. B. Ayres, Middlesex House, Department of Psychology, University of Massachusetts, Box 35530, Amherst, MA 01003-5530. lower middle and rear of the box. These changes in location were not seen when the tone and light CSs were explicitly unpaired with shock. Moreover, even though the forward procedure produced equivalent movement patterns and levels of conditioned suppression in the presence of auditory and visual CSs, freezing was greater to the auditory cue. The fact that tone and light CSs evoked similar withdrawal from the bar could explain how aversive auditory and visual cues produce similar barpress suppression, even when auditory cues evoke.more freezing.

The present experiments provide a more detailed examination of the relationship between change in location and conditioned suppression than did Bevins and Ayres's (1992) study.

\section{EXPERIMENT 1}

In Experiment 1, we departed from the prior work of Bevins and Ayres (1992) in three ways. First, we used a within-subject design to replicate the finding that a CS paired with shock (CS+) produces changes in location, whereas an unpaired CS (CS-) does not. This design provided a more efficient and sensitive assessment of the effects of conditioning procedure on location. Second, we used an A-B-A design to assess the effect of returning rats to on-baseline training following off-baseline training. This procedure enabled us to determine whether CS-evoked changes in location, which are eliminated by a shift from on-baseline to off-baseline procedures (Bevins \& Ayres, 1992), are restored when rats are returned to the original on-baseline procedure. Finally, we assessed the effects of training order by comparing the above design with a B-A-A design, in which rats first received off-baseline training and then received onbaseline training. 


\section{Method}

Subjects

The subjects were 16 male albino rats, 90 days old on arrival from the Holtzman Company, Madison, WI. They were housed singly in suspended stainless steel cages in a room maintained under constant illumination. All the rats first served as subjects in a onetrial, backward fear-conditioning experiment in another laboratory. Then, for the present experiment, they were reduced to and maintained at $80 \%$ of their free-feeding weights. The rats were 108 days old at the start of deprivation.

\begin{abstract}
Apparatus
Barpress training and aversive conditioning occurred in eight Gerbrands operant boxes, each measuring $23.2 \mathrm{~cm}$ long $\times 20.3 \mathrm{~cm}$ high $\times 19.5 \mathrm{~cm}$ wide. The end walls of each box were aluminum; the side walls and lid were clear Plexiglas. Centered in one end wall was a standard Gerbrands bar $(5 \times 1.5 \mathrm{~cm})$, located $8 \mathrm{~cm}$ above the floor. A recessed dipper tray $(5 \times 5 \times 5.5 \mathrm{~cm})$ was located in the lower left corner of the wall. During off-baseline training, a metal plate covered the bar and dipper. The plate reduced the inside length of the box by $1.7 \mathrm{~cm}$. The grid floor consisted of 18 ( $2 \mathrm{~mm}$ in diameter) stainless steel rods, spaced $1.3 \mathrm{~cm}$ apart center to center. Scrambled gridshock $(.8 \mathrm{~mA}$ for $1 \mathrm{sec})$, delivered to the floor from one of eight Grason-Stadler shock sources (Models E1064 or 700 ), served as the US. A $1000-\mathrm{Hz}, 86-\mathrm{dB}$ tone, delivered through a speaker located on the lid of each box, served as the auditory CS. Intensity was measured by using a General Radio Model 1565-B sound-level meter that was set on the " $\mathrm{C}$ "' scale with the microphone about $7 \mathrm{~cm}$ from the dipper. Each operant box was housed in a ventilated sound-attenuating enclosure. A red $7.5-\mathrm{W}$ bulb mounted on the rear wall of the enclosure provided constant background illumination at $2 \mathrm{~lx}$. A second, white 7.5 -W bulb on the upper rear wall served as the light CS. The combination of red and white bulbs during a CS presentation yielded $17 \mathrm{~lx}$. Each attenuating enclosure had a double-paned Plexiglas door that allowed full view of the rat. Observations during conditioning were paced by a relay and nine indicator lamps, located on a rack above the soundattenuating enclosures and outside of the rats' view.
\end{abstract}

\section{Procedure}

The rats were assigned to one of two groups ( $n=8$ per group), balanced for prior experimental history. Appetitive baseline training and Pavlovian fear conditioning occurred in the following phases.

Appetitive baseline training. After initial magazine training and response shaping, all the rats were trained for five $1-\mathrm{h}$ sessions on a variable interval (VI) 1-min schedule of reinforcement with a variable time 1-min limited hold. Barpressing was maintained throughout the experiment with 4 -sec access to a $.1-\mathrm{ml}$ dipper cup containing $32 \%$ liquid sucrose.

Phase 1. Phase 1 consisted of eight daily 1 -h sessions in which both groups received a 2-min tone CS, a 2-min light CS, and a .8-mA $(1-\mathrm{sec})$ scrambled gridshock US. For half of the rats in each group, the tone (CS+) coterminated with shock, and the light (CS-) was explicitly unpaired with shock; the modality of CS+ and CS- was reversed for the other rats in each group. For all the rats, the tone began at Minutes 19, 45, 24, and 38, respectively, on Days 1, 2, 3 , and 4; the light began at Minutes 47, 28, 40, and 20 during these days. This presentation sequence was repeated for Days 5-8 of Phase 1. The rats in Group On were allowed to barpress and consume sucrose during each of the eight sessions of Phase 1 (onbaseline fear conditioning). The rats in Group Off were not allowed to barpress or consume sucrose during this phase (off-baseline fear conditioning).

Phase 2. Phase 2 consisted of eight daily 1-h sessions. The aversive-conditioning parameters used in Phase 1 also were used during Phase 2 . The only difference between phases was that Group On was shifted to an off-baseline procedure, and Group Off was shifted to an on-baseline procedure.
Phase 3. Phase 3 consisted of 10 daily 1-h sessions of on-baseline conditioning for both groups. All other aspects of the aversive procedure were the same as those in Phase 1.

\section{Behavioral Observations}

We scored the location of each rat in the operant box by using a time-sampling procedure that yielded 10 observations per minute during each 2-min pre-CS, CS+, and CS- period (cf. Bouton \& Bolles, 1980; Fanselow \& Bolles, 1979; Sigmundi, Bouton, \& Bolles, 1980). Half of the rats in each group were scored on any given day; the remaining rats in each group were scored on the following day. Thus, we obtained one location measure for all the rats in a group on consecutive 2-day blocks of training.

We defined location by the position of a rat's nose at the time of an observation. Six cells $(7.7 \mathrm{~cm}$ wide $\times 10.1 \mathrm{~cm}$ high) were created by marking the Plexiglas wall that faced the observer with 1-mm-wide black tape. We arbitrarily numbered the top cells 1 , 2 , and 3 , and the bottom cells 4,5 , and 6 from the left to right of the observer. Cell 4 contained the response bar; the light CS was closest to Cell 2; and the tone speaker was mounted on the ceiling above Cell 3. During on-baseline training, location in the dipper tray was also scored. The dipper tray was labeled Cell 0. The size of Cells 1 and 4 was reduced by $1.7 \mathrm{~cm}$ by the metal plate that covered the bar and dipper during off-baseline training.

We report barpress suppression data only for on-baseline training phases and only for those days for which location was scored for a rat. Suppression was expressed as a ratio, formed by dividing the number of responses occurring during a CS by the total responses occurring during pre-CS and CS periods (Annau \& Kamin, 1961). $A$ value of .5 denotes no suppression to the $C S$, and a value of 0 denotes total suppression to the CS.

\section{Results}

\section{Barpress Suppression}

A preliminary analysis showed no difference in suppression to the tone and light when these cues were paired with shock in Phase $1[F(1,6)=2.94]$, and so the tone and light data are pooled in subsequent analyses and graphic presentations. (A two-tailed rejection region of .05 was adopted in all statistical analyses.) Figure 1 shows mean suppression ratios during the on-baseline training phases in Group On (top panels: Phases 1 and 3) and Group Off (bottom panels: Phases 2 and 3). The abscissa label (measure) refers to consecutive blocks of two sessions for which suppression and location data were available for all rats in a group.

The top left panel shows the acquisition of barpress suppression during Phase 1 in Group On. As training progressed, suppression increased to CS+ but not to CS-. A repeated measures analysis of variance (ANOVA; CS event [CS+ vs. CS -$] \times$ measure) yielded significant main effects of event $[F(1,7)=140.77]$ and measure $[F(3,21)=$ 16.43] and a significant event $\times$ measure interaction $[F(3,21)=11.62]$. The pattern of strong suppression to CS+ and weak suppression to CS- resumed and persisted in Phase 3 following interpolated off-baseline training in Phase 2. In Phase 3, the ANOVA showed only a significant effect of event $[F(1,7)=127.91]$.

The lower half of Figure 1 shows suppression ratios for Group Off during the on-baseline sessions of Phases 2 and 3. Suppression was strong to $\mathrm{CS}+$, but not to $\mathrm{CS}-$, in both phases. Repeated measures ANOVAs yielded a significant main effect of event $\left[F_{\mathrm{s}}(1,7)>201.2\right]$ and 


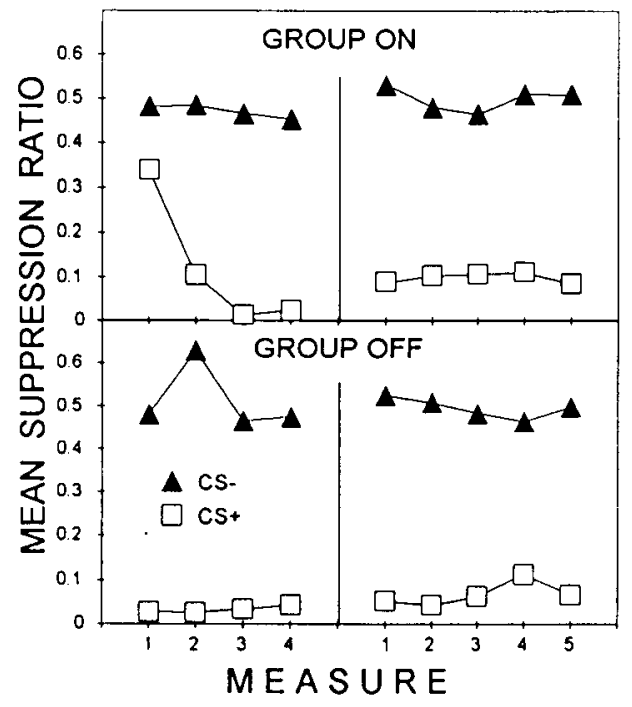

Figure 1. Barpress suppression in Experiment 1. Top: Suppression in Group On during on-baseline fear conditioning in Phase 1 (left panel) and Phase 3 (right panel). Bottom: Suppression in Group Off during on-baseline fear conditioning in Phase 2 (left panel) and Phase 3 (right panel).

a significant event $\times$ measure interaction in both phases ( $F \mathrm{~S}>3.25$ ), indicating suppression to $\mathrm{CS}+$ but not to CS-.

\section{Location}

Figure 2 shows the percentage of time that Group On spent in various parts of the box during Phases 1 (top panels), 2 (middle panels), and 3 (lower panels). The rats spent little time in the upper parts of the box (Cells 1 , 2 , and 3), so these observation cells have been eliminated from consideration. Additionally, Cells 5 and 6 (the lower middle and rear of the box) have been combined and are labeled "back," Cell 4 (the bar area) is labeled "bar," and Cell 0 is labeled "dipper."

There were no systematic changes in time spent in any of the areas during either $\mathrm{CS}-$ or pre-CS periods during Phase 1. However, over training, the time spent during $\mathrm{CS}+$ in the bar and dipper areas decreased, while time spent in the back of the box increased. Statistical analyses supported these impressions. Separate repeated measures ANOVAs (CS event [pre-CS, CS + , or $\mathrm{CS}-$ ] $\times$ measure), computed for each location, yielded a significant main effect of event for the dipper, bar, and back areas $[F \mathrm{~s}(2,14)>32.4]$. The main effect of measure $[F(3,21)=4.60]$ and the event $\times$ measure interaction also were statistically significant for the back area $[F(6,42)=4.50]$. Planned orthogonal contrasts indicated that CS- did not differ from the pre-CS period in terms of the percentage of time spent in any of the three areas. However, CS + differed significantly from CS - and pre$\mathrm{CS}$ combined for each of the three areas $[F \mathrm{~s}(1,7)>62.4]$. Thus, relative to pre-CS and CS- combined, CS+ reduced the time spent in the dipper and bar areas and in- creased the percentage of time spent in the back area. Relative to pre-CS, CS- produced no changes in time spent in these areas.

The middle panels of Figure 2 show location results for Group On during off-baseline training in Phase 2. The panel labeled "dipper" is empty because the dipper was inaccessible. There were no systematic changes in the time spent in the bar or back areas during CS,$+ \mathrm{CS}-$, or preCS events. Repeated measures ANOVAs yielded no statistically significant main effects or interactions.

The bottom panels of Figure 2 show location data for Group On when they were returned to on-baseline training in Phase 3, thus completing the A-B-A design. At the beginning of Phase 3, the location trends seen at the end of Phase 1 were only partly restored. The rats withdrew from the dipper but not from the bar area during $\mathrm{CS}+$. Moreover, movement to the back of the box was only partially renewed. However, as training progressed, the rats began spending less time in the bar area and more time in the back of the box during CS+. By the end of Phase 3, they behaved more like they had at the end of Phase 1, moving out of the bar and dipper areas and into the back of the box during CS+. Additionally, relative to pre-CS, CS- presentations produced a small decrease in the percentage of time spent in the dipper area. Sepa-

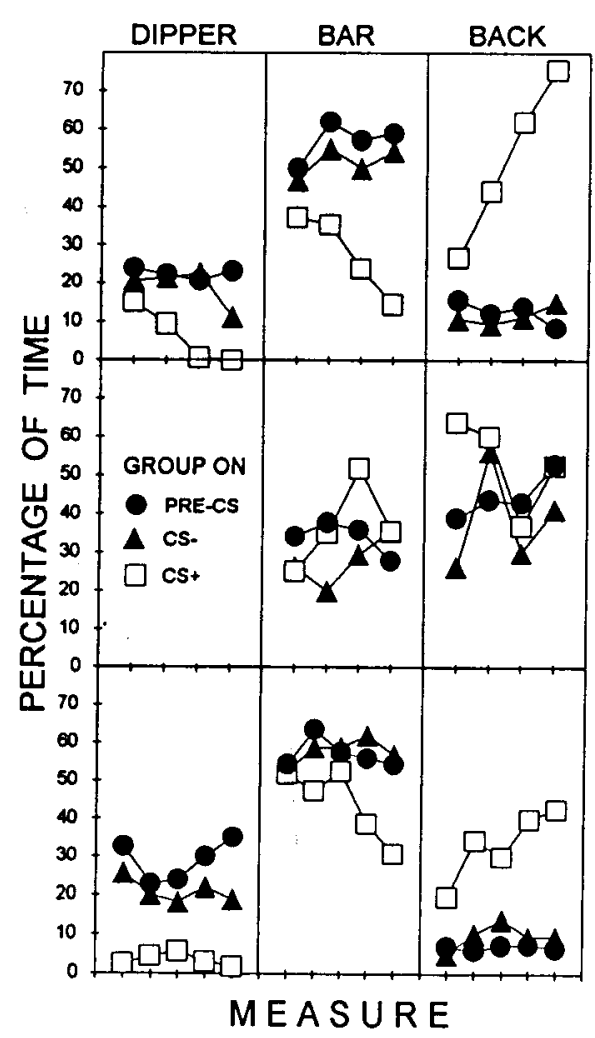

Figure 2. Time spent in given areas of the box for Group On in Experiment 1. Top: Phase 1 (on-baseline fear conditioning). Middle: Phase 2 (off-baseline fear conditioning). Bottom: Phase 3 (onbaseline fear conditioning). The plotted pre-CS data reflect the average of the pre-CS+ and pre-CS- periods. 
rate repeated measures ANOVAs (event $\times$ measure) yielded a significant main effect of event in all three areas $[F \mathrm{~s}(2,14)>5.09]$. There was a significant event $\times$ measure interaction in the dipper area $[F(8,56)=2.45]$. Planned orthogonal contrasts indicated that CS - differed from the pre-CS period only in the percentage of time spent in the dipper area of the box $[F(1,7)=15.13]$. In addition, CS+ differed significantly from the combined percentages for pre-CS and CS- periods for the dipper and back areas $\left[F_{\mathrm{s}}(1,7)>11.6\right]$; this contrast approached significance for the bar area $[F(1,7)=5.54, p=.0507]$.

Figure 3 shows the time spent in defined parts of the box for Group Off during all three phases of training. The top left panel contains no data because the dipper was inaccessible during the off-baseline procedure of Phase 1 . The remaining top panels show no systematic changes in time spent in the bar and back areas during CS + , CS - , or pre-CS periods. Indeed, separate repeated measures ANOVAs (event $\times$ measure) on each location yielded no significant main effects or interactions.

The middle panels of Figure 3 show the on-baseline location results for Group Off in Phase 2. Throughout training, the rats spent less time in the dipper area and, correspondingly, more time in the back area during CS+ relative to the pre-CS or CS- periods. This pattern was

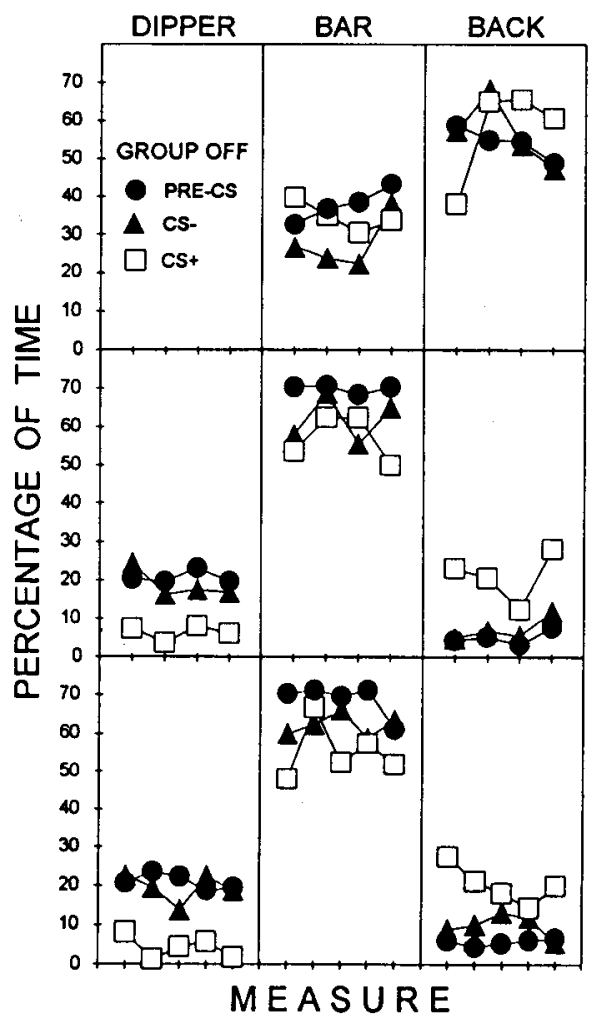

Figure 3. Time spent in given areas of the box for Group Off in Experiment 1. Top: Phase 1 (off-baseline fear conditioning). Middle: Phase 2 (on-baseline fear conditioning). Bottom: Phase 3 (onbaseline fear conditioning). The plotted pre-CS data reflect the average of the pre-CS+ and pre-CS- periods. evident from the outset of Phase 2 and thus did not develop gradually, as it had in Group On during the onbaseline sessions of Phase 1 (see the top panels of Figure 2). Separate repeated measures ANOVAs (event $x$ measure) for each location yielded a main effect of event for the dipper and back areas $[F \mathrm{~s}(2,14)>7.1]$, and the contrast comparing CS+ with CS- and pre-CS periods combined proved significant $[F \mathrm{~s}(1,7)>7.3]$. No systematic changes in time spent in the bar area during any of the event types were found.

The results in Phase 3 for Group Off are shown in the bottom panels of Figure 3. Phase 3 merely continued the on-baseline training of Phase 2, and the results of the two phases were similar with one minor exception: relative to the pre-CS period, the CS- slightly increased the time spent in the back of the box $[F(1,7)=18.03]$. Thus, the main finding in Phase 3 was that, even with extensive onbaseline training (18 CS-US pairings in Phases 2 and 3 combined), Group Off never developed the pattern of leaving the bar area during $\mathrm{CS}+$. That pattern characterized Group On during both their first training on baseline and their second training on baseline after intervening training off baseline.

\section{Within-Trial Location Changes}

Figure 4 shows the percentage of time that the rats spent in the dipper, bar, and back areas in four successive 30-sec bins of pre-CS+, CS+, and CS- periods. The data are from the last block of two sessions (Measure 4) of Phase 1 and reflect terminal performance in on- and off-baseline conditions for Groups On and Off, respectively. The top half of the figure shows the results for Group On and suggests that these rats spent an increasing amount of time in the back of the box over the course of the CS+ and, correspondingly, spent less time in the bar area. The bottom half of the figure shows the results for the rats in Group Off and suggests no systematic within-trial changes in location. The data in each panel of the figure were subjected to separate CS event $\times$ bin ANOVAs. Of main interest was a significant event $\times$ bin interaction, which occurred only for Group On and only in the back area $[F(6,42)=3.96]$. The meaning of this interaction was clarified by ANOVAs performed separately on the pre-CS +, CS +, and CS- data. The main effect of bin was found to be significant only during $\mathrm{CS}+[F(3,21)=$ 3.13].

\section{Discussion}

Several conclusions emerge from the results of this experiment. (1) The order of on- versus off-baseline training determines the pattern of location effects seen on baseline. Only the rats that were first trained on baseline showed the full pattern of location effects described by Bevins and Ayres (1992). (2) The factors responsible for withdrawal from the dipper tray seem to differ from those responsible for withdrawal from the bar area. All the rats came to withdraw from the dipper during CS+, but the rats given off-baseline training first never withdrew from the bar area. Moreover, the rats first trained on baseline 


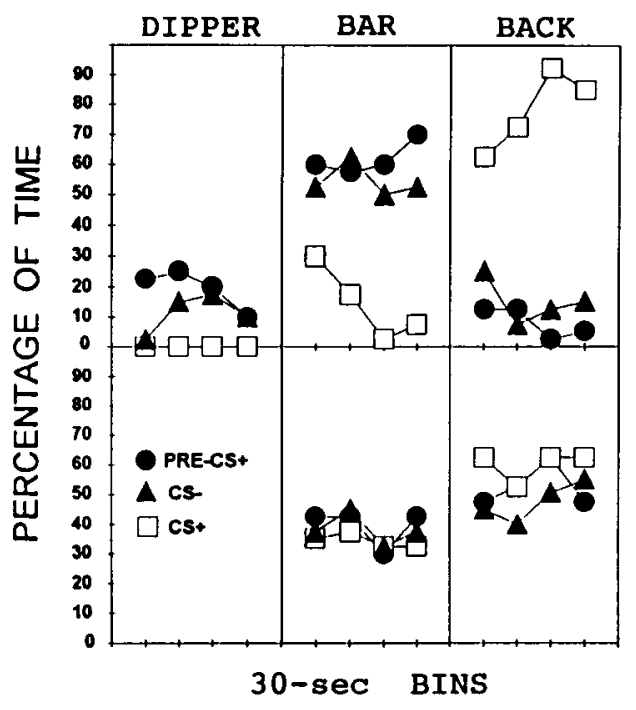

Figure 4. Time spent in the dipper, bar, and back areas of the box in four successive 30-sec periods (bins) of pre-CS+, CS + , and CS- periods in Experiment 1. Top panels: Group On. Bottom panels: Group Off.

increasingly withdrew from the dipper during CS+ as training progressed, but the rats first trained off baseline did so immediately when placed on baseline. (3) Barpress suppression appeared to be independent of the amount of time that the rats spent in the bar area. Thus, the rats in Group On withdrew from the bar area at the end of Phases 1 and 3, but not at the start of Phase 3; yet their barpress suppression was similar at all three times. It was also similar to that in Group Off, which never withdrew from the bar area. This result implies that withdrawal from the bar area may be a sufficient condition for conditioned barpress suppression, but it is not a necessary condition.

\section{EXPERIMENT 2}

In past work, location during initial on-baseline training has been measured for a maximum of eight CS-shock pairings. In Experiment 2, we extended that training to $23 \mathrm{CS}$-shock pairings (23 days of conditioning).

\section{Method}

\section{Subjects}

The subjects were 12 Holtzman-descended albino rats, 6 males and 6 females, bred in our colony. Females weighed from 299 to $385 \mathrm{~g}$, and males weighed between 501 and $585 \mathrm{~g}$. The rats were housed singly in suspended stainless steel cages and had 24-h access to water. All the rats first served as subjects in a one-trial context fear-conditioning experiment in another laboratory. For the present study, they were reduced to and maintained at $80 \%$ of their free-feeding weights. They were about 107 days old at the time of deprivation. For reasons unrelated to the present study, on Day 1 of barpress shaping, the rat colony was switched from continuous lighting to a 16:8-h light:dark cycle. The experiment was conducted during the light part of this cycle.

\section{Apparatus}

Unless noted, the apparatus was unchanged. Only four of the eight operant boxes were used. Background lighting was provided by a
7.5-W 110-V frosted white bulb. The white lamps used in Experiment 1 to pace observations were removed. Only the relay clicks served as pacing stimuli.

\section{Procedure}

Preliminary shaping and VI training were the same as in Experiment 1 except that, beginning with VI training, all sessions were $40 \mathrm{~min}$ long rather than $1 \mathrm{~h}$. After VI training, all the rats received 23 days of on-baseline conditioning. On each day, a single 2-min tone CS $(1000 \mathrm{~Hz}$ at $86 \mathrm{~dB})$ coterminated with a gridshock US $(.8 \mathrm{~mA}$ for $1 \mathrm{sec})$. The tone began at the start of Minutes 12,32 , and 22, respectively, on Days 1,2 , and 3 . This sequence was repeated for every 3-day block throughout training. The timesampling procedure of Experiment 1 was used again to score location for all the rats on Days $2,4,6,8,12,16,20,21,22$, and 23 of conditioning. To provide a check on the reliability of scoring, a second trained observer scored $80 \%$ of the observations; $87 \%$ of the scores obtained by the two observers matched exactly.

\section{Results and Discussion}

Initial analyses showed no difference in barpress suppression between females and males $(t<1)$, and inspection of the location data also suggested no gender differences. Accordingly, the data for males and females were combined. On Day 1, the mean barpress suppression ratio to the tone CS+ was .45. That ratio decreased to .07 by Day 3 and stayed around that level for the rest of conditioning.

Figure 5 shows the percentage of time that the rats spent in the dipper, bar, and back areas of the box during conditioning. As in Experiment 1 and in Bevins and Ayres (1992), the rats spent little time in the upper part of the box, and so these cells are not depicted.

Days 2, 4, 6, and 8 of Figure 5 show the changes in location typically seen with initial on-baseline training (cf. top panels of Figure 2). By Day 4, CS+ evoked movement out of the bar and dipper areas and into the back of the box. However, on Days 12 and 16 the tendency to move from the bar area to the back area weakened. Finally, on Days 20-23, movement from the bar area to the back was restored. In short, withdrawal from the bar area was transient. Note that the trend seen in the bar area was not seen in the dipper. Withdrawal from the dipper was permanent. CS event $x$ day ANOVAs showed a main effect of CS event in all the areas depicted $\left[F_{\mathrm{S}}(1,11)>\right.$ 8.6] and a main effect of day in the bar and back areas $[F \mathrm{~s}(9,99)>2.6]$. There was also a significant event $X$ day interaction in the bar and back areas $[F \mathrm{~s}(9,99)>$ 4.0].

Trend tests confirmed the transience seen visually in the bar and back areas. During the CS+ in both areas, there was a significant cubic and quartic component $[F \mathrm{~s}(1,109)>10.3]$. No trend was significant in the dipper area.

\section{GENERAL DISCUSSION}

These studies identify two factors that affect rats' location during conditioned suppression training. The first is the order in which on-baseline versus off-baseline training is given. The second is the length of initial on-baseline 


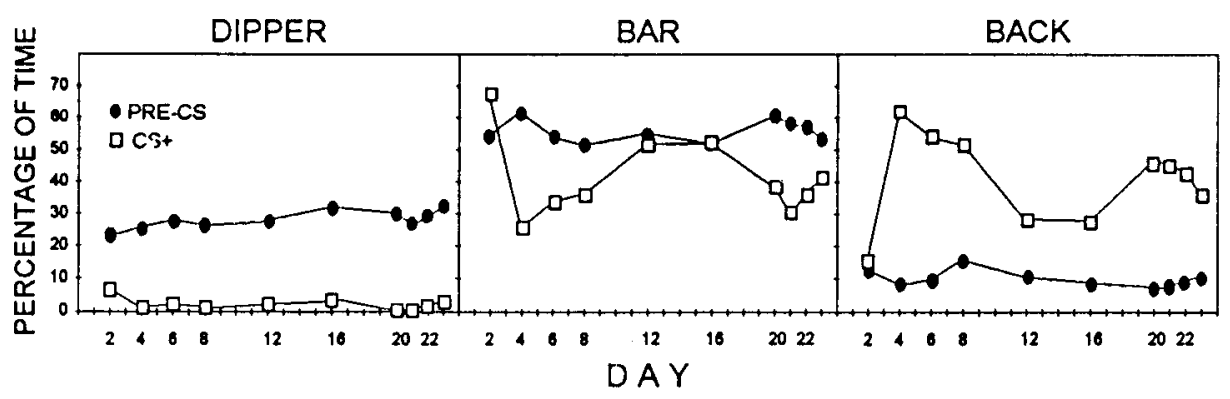

Figure 5. Time spent in given areas of the box during fear conditioning in Experiment 2.

training. The evidence can be summarized as follows. In Experiment 1, when on-baseline training occurred first, the rats gradually acquired the behavior of withdrawing from the dipper and bar areas and moving toward the middle and rear of the box during a CS paired with shock. Switching the rats to an off-baseline conditioning procedure disrupted that behavior immediately. When onbaseline training was resumed, withdrawal from the recessed dipper tray was immediate and strong, but withdrawal from the bar area was only gradually reacquired over sessions. When off-baseline training occurred first, the rats showed no systematic changes in location during CSs. Switching these rats to an on-baseline conditioning procedure produced immediate and profound CS-evoked withdrawal from the recessed dipper tray, but produced no withdrawal from the bar area even after extensive training ( 18 days at one CS + trial per day). In Experiment 2, the rats given on-baseline Pavlovian training from the outset came to withdraw from the bar and dipper areas during CSs paired with shock. With further Pavlovian conditioning, however, they stopped withdrawing from the bar area temporarily and then resumed withdrawing with further training. In short, withdrawal from the bar area appeared to be transient or even cyclic. Withdrawal from the recessed dipper tray, in contrast, was not. Once acquired, it remained strong throughout training.

Bevins and Ayres (1992) suggested several possible explanations for the changes in location that they observed during conditioned suppression training. The present results help us evaluate the merits of those explanations.

One explanation offered was that frightened rats prefer the odors of conspecifics. Rats trained on baseline tend to defecate in the rear of the box. Therefore, when frightened by a CS paired with shock, they withdraw to that area, where the odors are strongest. That explanation appears to be refuted by the present findings. First, the rats trained on and off baseline in Phase 1 of Experiment 1 were exposed to the same box and litter tray (which was never changed between groups and rarely changed between sessions). The odors in the box should have been similar for the two groups, yet their behaviors clearly differed: During CS+, the rats trained on baseline withdrew to the back of the box, but those trained off baseline did not. Similarly, when Group On was shifted from on- baseline training to off-baseline training, their behavior changed immediately - too fast, presumably, to be explained in terms of changing odors from one day to the next. Second, in Experiment 2, the rats' withdrawal from the bar area was cyclic despite the fact that the litter tray was never emptied.

A second explanation that appears to be refuted by the present data is that withdrawal from the bar and dipper areas minimizes the occurrence of behaviors that would compete with activities or postures that reduce the effectiveness of shock. These activities or postures should not lose their effectiveness with extended training. Therefore, this hypothesis has trouble explaining the transience or cyclicity of withdrawal from the bar area in Experiment 2.

During the editorial process, it was suggested that appetitive contrast might be invoked to explain why, in Experiment 1 , the rats were reluctant to withdraw from the bar following a shift from off-baseline training to onbaseline training. The assumption is that blocking a barpress-trained rat from the bar by a barrier increases the rat's reluctance to leave the bar when the barrier is removed. This idea fails to explain, however, why the rats trained off baseline first and then switched to onbaseline training were much more reluctant to withdraw from the bar than were the rats trained on baseline, then off baseline, and finally on baseline again. In addition, the idea of appetitive contrast offers no explanation for the transience or cyclicity of withdrawal from the bar area in Experiment 2.

Bevins and Ayres (1992) considered two ideas that seem capable of explaining all of the bar-withdrawal behavior seen in the present studies. One is the idea of discriminated adventitious punishment. According to a punishment account, the rat initially trained on baseline is likely to be in the bar area when shock occurs at the end of the CS. The rat is thus punished in the presence of that CS for being in that area. By staying in the back of the box on later CS trials, the rat passively avoids the punished behavior. The second idea involves the concept of conditioned place aversion, for which the CS acts as an occasion setter. Like the punishment explanation, the placeaversion account holds that the rat is likely to be in the bar area when first shocked, so this area becomes aversive during the CS. The present work cannot distinguish 
experimentally between the adventitious punishment and the place-aversion accounts. We believe that either can explain all of the bar-withdrawal behavior seen in these experiments. To illustrate, we shall arbitrarily choose the place-aversion account and use it to explain the results.

In Experiment 1, when the rats initially trained on baseline were shifted to off-baseline training, their barwithdrawal behavior was immediately disrupted. The reason is that they were less likely to be in the bar area at CS onset, because they could not earn food there and were physically blocked from the bar. They were more likely to be in other areas and, at shock onset, to be focusing on cues outside the bar area. This experience would make these other areas and cues aversive. When returned to onbaseline training, the rats would be slow to withdraw from the bar area because other areas were now aversive. The rats trained off baseline before being shifted to on-baseline training should be even slower to withdraw from the bar area when shifted for the first time to on-baseline training. They were very likely to have been in areas other than the bar area when first shocked, and these areas would have become aversive. When these rats were switched to on-baseline training, the aversive cues in these areas might well have blocked conditioning to the cues in the bar area (Kamin, 1968). Such a blocking effect could explain why the rats that received off-baseline training before on-baseline training never acquired the behavior of withdrawing from the bar, even after extensive training on baseline. Note that the blocking mechanism would not be expected to operate in the group that received on-baseline training first. That is, cues in the bar area should not have blocked conditioning to cues in the back, in part because the bar area cues were changed in Phase 2 by the insertion of the metal plate. Similarly, the cues in the back of the box should not have blocked conditioning to the cues in the bar area in Phase 3 for this group, because those bar cues already had a history of reinforcement in Phase 1.

In Experiment 2, the rats given extensive on-baseline training would first be shocked in the bar area, making it aversive. Later in training, they would likely be in the middle or back of the box and focusing on cues there when shocked, making these areas aversive during the CS. If they subsequently avoided these areas during later CSs, they would be shocked in the bar area again, making it more aversive and encouraging movement toward the middle or rear of the box. Thus, withdrawal from the bar area should be transient or cyclic.

Although both the punishment and place-aversion accounts offer some understanding of withdrawal from the bar area, none of the explanations we have considered explain the profound withdrawal from the recessed dipper tray. That withdrawal does not seem to be due to the same factors as the withdrawal from the bar area. We wonder if withdrawal from a nonrecessed feeding trough would be so profound.
When Bevins and Ayres (1992) found that light and tone CSs evoked similar withdrawal from the bar area, they suggested that this similar withdrawal could explain the similar barpress suppression evoked by those cues despite the greater freezing evoked by the tone. That suggestion rested on the fact that withdrawal from the bar area is a sufficient condition for barpress suppression. The present results do not challenge that suggestion, but they do clearly establish that withdrawal from the bar area is not a necessary condition for barpress suppression. There are two strong pieces of evidence for that conclusion. First, when the rats initially trained on baseline in Experiment 1 were switched to off-baseline training and then back to onbaseline training, they did not withdraw immediately from the bar area in this final phase. Even so, their barpress suppression remained just as strong as it had been when they were withdrawing from the bar area during the first on-baseline training phase. Second, in Experiment 2, the rats came to withdraw from the bar area, then stopped doing so, then began withdrawing again as training continued. Meanwhile, their barpress suppression remained constant as withdrawal from the bar area waxed and waned.

\section{REFERENCES}

ANNAU, Z., \& Kamin, L. J. (1961). The conditioned emotional response as a function of intensity of the US. Journal of Comparative \& Physiological Psychology, 54, 428-432.

Ayres, J. J. B., Axelrod, H., Mercker, E., Muchnik, F., \& VIGORITO, M. (1985). Concurrent observations of barpress suppression and freezing: Effects of CS modality and on-line vs. off-line training upon posttrial behavior. Animal Learning \& Behavior, 13, 44-50.

Ayres, J. J. B. , \& Vigorito, M. (1984). Posttrial effects of presenting vs. omitting expected shock USs in the conditioned suppression procedure: Concurrent measurement of barpress suppression and freezing. Animal Learning \& Behavior, 12, 73-78.

Bevins, R. A., \& Ayres, J. J. B. (1991). Two issues in Pavlovian fear conditioning: Selective fear of bright vs. dark, and CS determinants of CR form. Behavioural Processes, 24, 211-218.

BEVINS, R. A., \& AYRES, J. J. B. (1992). Rats' location during conditioned suppression training. Animal Learning \& Behavior, 20, 8-16.

Bouton, M. E., \& Bolles, R. C. (1980). Conditioned fear assessed by freezing and by the suppression of three different baselines. Animal Learning \& Behavior, 8, 429-434.

Estes, W. K., \& SkINNER, B. F. (1941). Some quantitative properties of anxiety. Journal of Experimental Psychology, 29, 390-400.

Fanselow, M. S., \& Bolles, R. C. (1979). Naloxone and shock-elicited freezing in the rat. Journal of Comparative \& Physiological Psychology, 93, 736-744.

Fanselow, M. S., \& Lester, L. S. (1988). A functional behavioristic approach to aversively motivated behavior: Predatory imminence as a determinant of the topography of defensive behavior. In R. C. Bolles \& M. D. Beecher (Eds.), Evolution and learning (pp. 185-212). Hillsdale, NJ: Erlbaum.

KamIN, L. J. (1968). "Attention-like"' processes in classical conditioning. In M. R. Jones (Ed.), Miami Symposium on the Prediction of Behavior: Aversive stimulation (pp. 9-31). Coral Gables, FL: University of Miami Press.

Sigmundi, R. A., Bouton, M. E., \& Bolles, R. C. (1980). Conditioned freezing in the rat as a function of shock intensity and CS modality. Bulletin of the Psychonomic Society, 15, 254-256. 